\title{
Black Holes as Markers of Dimensionality
}

\author{
Szymon Łukaszyk
}

Independent Scholar

szymon@patent.pl

\begin{abstract}
Black hole temperature $T_{B H}=T_{P} / 2 \pi d$ as a function of its Planck length diameter multiple $d$ is derived from black hole surface gravity and Hawking temperature. It is conjectured that this multiple corresponds to dimensionality of the graph of nature with $d=1 / 2 \pi$ describing primordial Big Bang singularity $\left(T_{B H}=T_{P}\right)$. A black hole interacts with the environment and observable black holes have uniquely defined Delaunay triangulations with a natural number of spherical triangles having Planck areas (bits), where a Planck triangle is active and has gravitational potential of $-c^{2}$ if all its vertices have a black hole gravitational potential of $-c^{2} / 2$ and is inactive otherwise. Temporary distribution of active triangles on an event horizon tends to maximize Shannon entropy. Black hole blackbody radiation, informational capacity fluctuations, and quantum statistics are discussed. On the basis of the latter, wavelength bounds for BE, MB, and FD statistics are derived as a function of a black hole diameter. A similarity of the logistic function and black hole FD statistics leads to the $\mathrm{BE}$ logistic function and map. The program for research of other nature phenomena that emit perfect blackbody radiation, such as neutron stars and white dwarfs is outlined.
\end{abstract}

Keywords: quantum black holes, entropic gravity; the black hole information paradox; Shannon entropy; Delaunay triangulation; black hole quantum statistics; logistic function/map; exotic $\mathbb{R}^{4}$

\section{Introduction}

The combinatorial proof of the $H$-theorem [1] that Ludwig Boltzmann derived in 1877 introduced energy quantization. This led to the development of quantum theory [2] and brought about to the problem of measurement, performed hic et nunc by any particular observer, yielding rational information. This information is twofold; it relates both to without of things, as well as to within of things; quoting Pierre Teilhard de Chardin "In the eyes of the physicist, nothing exists legitimately, at least up to now, except the without of things. The same intellectual attitude is still permissible in the bacteriologist, whose cultures (apart from some substantial difficulties) are treated as laboratory reagents. But it is already more difficult in the realm of plants. It tends to become a gamble in the case of a biologist studying the behavior of insects or coelenterates. It seems merely futile with regard to the vertebrates. Finally, it breaks down completely with man, in whom the existence of a within can no longer be evaded, because it is the object of a direct intuition and the substance of all knowledge" [3].

\section{Spacetime Dimensional, Dimensional, and Adimensional}

Dimensionalities of the within (the interior) and the without (the exterior) of things are researched in this paper. The $(n+1)$-dimensional interior is bounded by $n$-dimensional topological sphere as asserted by the Jordan-Brouwer separation theorem. A black hole is an exception: unbounded black hole exterior has a boundary (event horizon) but a black hole does not have a bounded interior. Therefore black hole invalidates not only the Jordan-Brouwer separation theorem but also the generalized Stokes theorem: a differential form over the black hole event horizon is not equal to the integral of its exterior derivative over the whole of black hole. There is no such thing as the whole of black hole.

Every simplicial $n$-manifold inherits a natural topology from $\mathbb{R}^{n}$ [4] and by researching Euclidean space $\mathbb{R}^{n}$ as a simplicial $n$-manifold (made of triangles, tetrahedrons, etc.) topological (metric-independent) and geometrical (metric-dependent) content of the 
modeled quantities are disentangled [4]. This disentanglement is perhaps the most important virtue of the simplicial formulation.

Author shares the it from bit John Archibald Wheeler conclusion, yet is of the opinion that his statement that "there is no such thing at the microscopic level as space or time or spacetime continuum" [5] remains valid at any level, not only microscopic one. Nature should be researched as a vertex-labeled graph (graph of nature) having certain intrinsic properties reflecting the $2^{\text {nd }}$ law of thermodynamics. Primordial Big Bang singularity (the first point) expanded into dimensionalities [6] not into a 4-dimensional spacetime.

Dimension of a space equals a minimum number of independent parameters (coordinates) needed to specify a point within this space. Spaces with natural and low dimensionalities are the most interesting: Fermat's Last Theorem indicates the importance of $n \leq 2 ; n=4$ models spacetime, maximizes the number (six) of regular polytopes (excluding $n \neq 2$ ) and has the exotic $\mathbb{R}^{4}$ property; unit radius $n$-ball attains maximum volume for $n=5$ and maximum surface for $n=7$, and so on. Dimensionality of the graph of nature is by no means restricted to natural numbers. Negative and fractal dimensions can be successfully applied in the fields of diffusion-limited aggregation and turbulence [7] for example. However, since a point in fractionally dimensional space cannot be unambiguously defined within this space, such a point cannot be observed. Such negative, fractional and imaginary dimensionalities remain latent (=hidden, but present) [7]. For humans latent dimensionalities obviously also include $n>3$.

In the following the term "spacetime dimensional" refers to spacetime dimensionality $\left(\mathbb{R}^{4}\right)$; the term "dimensional" has a broader meaning and refers both to $\mathbb{R}^{4}$, as well as other natural dimensionalities $n \in \mathbb{N}$; the term "fractionally dimensional" has an even broader meaning and refers both to $n \in \mathbb{N}$ o and to $n \in \mathbb{N} / \mathbb{R}$ defining fractional dimensionalities; and finally the term "adimensional" refers both to $n \in \mathbb{R}$, as well as to the remaining negative and imaginary dimensionalities. The notion of charge and other already unified quantities should, in the author's opinion, emerge from topological and entropic considerations alone [8]. Entropy is certainly adimensional.

\section{Black Holes and Dimensionality}

The fundamental black hole relation $R_{B H}=2 G M / c^{2}$ seems to be fractionally dimensional. Commonly called the Schwarzschild radius to honor Karl Schwarzschild solution to Einstein's field equations for a spherically symmetric body with a mass $M$, this simple formula also stems from the escape velocity equality $2 G M / R_{B H}=c^{2}$. Thus it seems reasonable to assume that it remains valid in any fractional dimension. Interestingly and without loss of generality by expressing black hole mass as $M:=m m P, m \in \mathbb{R}$, $m P$ denoting Planck mass, the Schwarzschild radius becomes

$$
R_{B H}=\frac{2 G m m_{P}}{c^{2}}=\frac{2 m G}{c^{2}} \sqrt{\frac{\hbar c}{G}}=2 m \ell_{P} .
$$

The holographic principle [9, 10,5] assumes spacetime dimensionality of the bounded interior and unbounded exterior separated by 2-dimensional boundary having two spatial dimensions and time dimension. Having an area $A$, the boundary has an informational capacity $N_{A}=A / \ell_{P}^{2} \in \mathbb{R}$ with one bit represented by one Planck area $\ell_{P}^{2}$. Therefore the number of bits the boundary provides is $\left\lfloor N_{A}\right\rfloor \in \mathbb{N}$. Black hole event horizon is a fundamental holographic boundary having only one geometric side observable as a 2-sphere. Without loss of generality we can define the diameter of this sphere as $D:=d \ell_{P}, d \in \mathbb{R}$, so that the informational capacity of the event horizon is $N_{B H}=\pi D^{2} / \ell_{P}^{2}=\pi d^{2}$. Plugging black hole surface gravity into Hawking radiation formula one arrives at the Hawking radiation temperature as a function of $N_{B H}$ or diameter multiples $d$ 


$$
T_{B H}=\frac{m_{P} c^{2}}{2 k_{B} \sqrt{\pi N_{B H}}}=\frac{T_{P}}{2 \sqrt{\pi N_{B H}}}=\frac{T_{P}}{2 \pi d},
$$

as illustrated in Fig. 1, where $T_{P}$ denotes Planck temperature (cf. Appendices). Since no physical models describe temperatures greater than the Planck temperature, the primordial Big Bang singularity temperature corresponds to a black hole having diameter $d=1 /(2 \pi), N_{B H}=1 /(4 \pi)$ and $\left[N_{B H}\right]=0$. At $d=1$ black hole has temperature is $T=T_{P} / 2 \pi$, which can be regarded as a reduced Planck temperature similarly as the reduced Planck constant or a reduced wavelength.

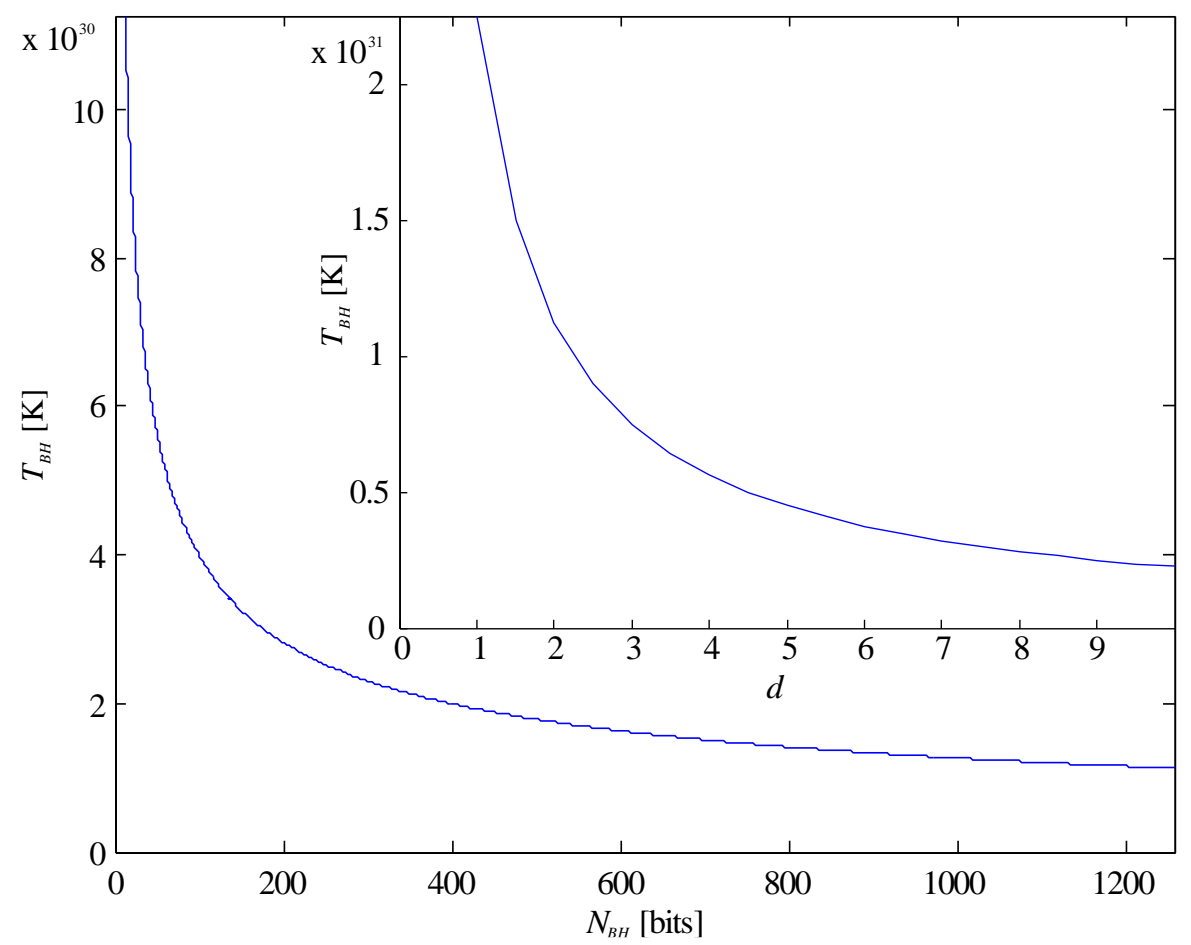

Fig. 1: Black hole temperature $T_{B H}$ as a function of its informational capacity $N_{B H}$ and diameter multiple $d$.

Natural multiples $d$ of black hole diameter are related to $2 \pi$ cycle: black hole temperature (2) decreases by $2 \pi$ factor with every integer increment of $d$. Thus $d$ seems to be related to the dimensionality of the graph of nature.

Table 1 is a list of a few quantum black holes showing informational capacity, number of bits, gravitational potential with phasor $\theta$ set to 1 [8]

$$
\delta \varphi=-\frac{4 \pi c^{2}}{N_{B H}} \frac{\delta r}{\hbar}=-\frac{4 \pi c^{2}}{N_{B H}} \theta,
$$

mass and temperature of each hole as a function of its diameter multiple $d$.

Black holes with $d<1$ correspond to a point, $\pi$-bit black hole $(d=1)$ corresponds to a curve, and $4 \pi$-bit black hole $(d=2)$ corresponds to a surface. Diameter multiple $d=2 \sqrt{ } 2 \approx 2.83$ provides gravitational potential (3) equal to $-c^{2} / 2$ and the mass of $16 \pi$-bit black hole $(d=4)$ corresponds to Planck mass $m_{P}$ [8]. Any real black hole such as Sagittarius $\mathrm{A}^{*}\left(M_{B H} \approx 8.62 \mathrm{e} 36 \mathrm{~kg}, d \approx 1.58 \mathrm{e} 45, N_{B H} \approx 7.88 \mathrm{e} 90\right)$ does not exist as an object in spacetime but is only observable as 2 -sphere by observers that can only exist in four dimensions [6].

The number of bits is clearly a natural number (OEIS A066643), while the black hole informational capacity $\left(\pi d^{2}\right)$ is a transcendental number. Perhaps nature knows how to distinguish a set of vertices of a given black hole from a set of vertices of another one. 
This certainly requires further research. Interestingly a fractional part has Fourier series expansion [11], so that

$$
\left\{N_{B H}\right\}=\left\{\pi d^{2}\right\}=\frac{1}{2}-\frac{1}{\pi} \sum_{k=1}^{\infty} \frac{\sin \left(2 k \pi^{2} d^{2}\right)}{k}
$$

Table 1: Quantum black holes and dimensionality of the graph of nature

\begin{tabular}{|c|c|c|c|c|c|c|}
\hline$d=D / \ell_{P}$ & $N_{B H}=\pi d^{2}$ & $\left\lfloor\pi d^{2}\right\rfloor$ & $\begin{array}{c}\delta \varphi / c^{2}= \\
-4 / d^{2}\end{array}$ & $\begin{array}{c}M_{B H} / m_{P}= \\
d / 4\end{array}$ & $\begin{array}{c}T_{B H} / T_{P}= \\
1 /(2 \pi d)\end{array}$ & black hole type/comments \\
\hline $1 /(2 \pi)$ & 0.0796 & 0 & $-16 \pi^{2}$ & 0.0398 & 1 & Big Bang (Planck temperature) \\
\hline $1 / \sqrt{ } \pi$ & 1 & 1 & $-4 \pi$ & 0.1410 & 0.2821 & min 1-bit, $\mathrm{MB}$ stat. singularity \\
\hline$\sqrt{ }(\ln (4) / \pi)$ & 1.3863 & 1 & -9.0647 & 0.1661 & 0.2396 & Landauer $\mathrm{BH}\left(M_{B H C^{2}}=T k_{B} \ln (2)\right)$ \\
\hline$\sqrt{ }(2 / \pi)$ & 2 & 2 & $-2 \pi$ & 0.1995 & 0.1995 & min 2-bit, FD stat. singularity \\
\hline$\sqrt{ }(3 / \pi)$ & 3 & 3 & -4.1888 & 0.2443 & 0.1629 & $\min 3$-bit \\
\hline 1 & $\pi$ & 3 & -4 & 0.25 & 0.1592 & $\pi$-bit \\
\hline$\sqrt{ }(4 / \pi)$ & 4 & 4 & $-\pi$ & 0.2821 & 0.1410 & min 4-bit, one unit of entropy \\
\hline 2 & $4 \pi$ & 12 & -1 & 0.5 & 0.0796 & 12-bit \\
\hline $2 \sqrt{ } 2$ & $8 \pi$ & 25 & -0.5 & $1 / \sqrt{ } 2$ & 0.0563 & potential equal to $-G M / R_{B H}=-c^{2} / 2$ \\
\hline 3 & $9 \pi$ & 28 & $-0.4(4)$ & 0.75 & 0.0531 & 28-bit \\
\hline 4 & $16 \pi$ & 50 & -0.25 & 1 & 0.0398 & $M_{B H}=m_{P}$ \\
\hline 5 & $25 \pi$ & 78 & -0.16 & 1.25 & 0.0318 & \\
\hline $8 \pi$ & $64 \pi^{3}$ & 1984 & -0.0063 & $2 \pi$ & 0.0063 & $M_{B H}=2 \pi m P\left(\delta \varphi / c^{2}+T_{B H} / T_{P}=0\right)$ \\
\hline
\end{tabular}

\section{Delaunay Triangulated 2-Boundaries}

Only Delaunay triangulation of the set of vertices $V$ defining 2-dimensional boundary minimizes the Dirichlet energy [12]

$$
E_{D}[\varphi] \doteq \frac{1}{2} \varphi^{T} L \varphi
$$

of any piecewise linear function $\varphi: V \rightarrow \mathbb{R}$ over this point set (Rippa's theorem [13]), where $L$ is the discrete cotan Laplacian. This is equal to say that the spectrum of the cotan Laplacian obtains its minimum on a Delaunay triangulation in the sense that the $i$-th eigenvalue of the cotan Laplacian of any other triangulation is bounded below by the $i$-th eigenvalue of the cotan Laplacian of the Delaunay triangulation [14]. Only Delaunay triangulation of a piecewise flat surface guarantees that edge weights of the cotan Laplacian are positive [15].

At least due to these properties it seems reasonable to assume that Delaunay triangulations would be used by nature to create equipotential surfaces as holographic boundaries, where each triangle has at most one Planck area $\ell_{P}^{2}$ representing 1 bit of rational information. Thus Delaunay triangulated $\mathbb{R}^{2}$ boundary is equivalent to its continuous counterpart [4] and $\varphi$ in (5) represents a harmonic potential assigned to vertices of the triangulation.

It seems further reasonable to assume that any triangle on the boundary is a geodesic, non-planar one. A surface made of planar and geodesic triangles might not be twice differentiable as required by Laplace and Poisson's equations. Only Delaunay circles make the boundary "piecewise flat". A stronger version of this assumption would be to demand that all boundaries are topological spheres and differ only with radii and 
uniqueness or the rank of non-uniqueness (i.e. the number of cyclic spherical quadrilaterals) of the triangulation.

Expressing the variation of entropy $\delta S$ on an equipotential 2-dimensional surface $A$ [16, eq. (1) $\left.{ }^{1}\right]$ (which is equivalent to the holographic boundary)

$$
\delta S=-\frac{c k_{B}}{2 G \hbar} \delta \varphi A,
$$

where $\delta \varphi$ represents a variation of the potential on the boundary, as a function of the boundary informational capacity we arrive at

$$
\delta S=-\frac{c k_{B}}{2 G \hbar} \delta \varphi N_{A} \ell_{P}^{2}=-\frac{1}{2} k_{B} N_{A} \frac{\delta \varphi}{c^{2}} .
$$

This equation can be simplified by postulating a binary variation of the potential (or simply a binary potential) in Planck time $t_{P}$ over $k$-th Planck triangle $\ell_{P}^{2}$ defined as

$$
\delta \varphi_{k} \doteq-\{0,1\} \ell_{P}^{2} / t_{P}^{2}=-\{0,1\} c^{2},
$$

so that

$$
\delta S=-\frac{1}{2} k_{B} \sum_{k=1}^{\left\lfloor N_{A}\right\rfloor} \frac{\delta \varphi_{k}}{c^{2}}=\frac{1}{2} k_{B} N_{1},
$$

where the summation goes over all Planck triangles of the boundary $A, N_{1} \in \mathbb{N}$ denotes the number of Planck triangles that encode ${ }^{2}-c^{2}$, and triangle(s) of the fractional part $\left\{N_{A}\right\}$ of the boundary informational capacity encode(s) zero [8].

In this setting one Planck triangle that encodes $-c^{2}$ can be taken to represent one degree of freedom, so that the equipartition theorem

$$
E=T \delta S=\frac{1}{2} k_{B} T
$$

is recovered.

One vertex may be shared by many triangles, two vertices by two triangles. Only all three vertices of a triangle having the same potential of $-c^{2} / 2$ provide unique association of $-c^{2}$ to this triangle. Therefore "active" vertex has gravitational potential of $-c^{2} / 2$; "inactive" vertex has gravitational potential higher than $-c^{2} / 2$ (diameter fluctuates, due to black hole absorption and emission). Planck triangle is "active" if all its vertices are active and then has gravitational potential of $-c^{2}$; otherwise it is "inactive" and encodes zero. Fractional part triangle(s) always encode(s) zero and its/their total area is lower than Planck area.

A black hole event horizon should have a uniquely defined Delaunay triangulation with at least one spherical triangle having an area corresponding to the fractional part of its informational capacity $\left\{N_{B H}\right\}=N_{B H}-\left[N_{B H}\right]$, and all the remaining triangles having Planck areas. Uniquely defined means that no four vertices on the event horizon share a common circle [17], which is equal to say that no two triangles on the event horizon form a cyclic spherical quadrilateral. Two regular (uniquely defined) Delaunay triangles have $N_{B H}=2$ (and two circumcenters), but two equally possible orientations of a diagonal on a cyclic spherical quadrilateral (in particular on a spherical rectangle) having vertices, say 1

\footnotetext{
1 The additive constant in this equation may be taken to represent the fractional part $\left\{N_{A}\right\}$ of the holographic boundary informational capacity.

2 Binary potential was originally defined as a positive quantity. This is, however, in conflict with the negative value of the gravitational potential at a vertex of the event horizon $\left(-c^{2} / 2\right)$. Anyway the sign seems irrelevant in calculating statistics of bits on the boundary.
} 
to 4 , and only one circumcenter would enable to assign an additional bit to this pair of triangles. Certainly triangles $\{1,2,3\}$ and $\{1,3,4\}$ are different than triangles $\{1,2,4\}$ and $\{2,3,4\}$ even if in both cases they would encode $-c^{2}$. Non-uniquely defined Delaunay triangulation would invalidate the Bekenstein bound. Non-unique triangulation enables formation of dissipative structures [18] that operate out of thermodynamic equilibrium. But a black hole is a thermodynamic equilibrium. A fundamental one.

Vertices define inner black hole polyhedrons that in turn define black hole cotan Laplacians and have area smaller than the area of the circumscribed sphere. The ratio of these areas rapidly converges toward unity as the size of the black hole increases. Therefore large black holes are observable as isotropic and thus stable, while the small ones quickly evaporate [20].

It turns out that the temporary distribution of active triangles on an event horizon of a black hole tends to maximize Shannon entropy [8]. This results from comparing the entropic works on the event horizon with Hawking temperature and Bekenstein-Hawking entropy or the variation of binary entropy (9)

$$
\begin{aligned}
& T=-\frac{\hbar}{2 \pi c k_{B}} \nabla_{R} \phi_{g} \\
& T \delta S=\left(-\frac{\hbar}{2 \pi c k_{B}} \frac{G M}{\frac{N_{B H} \ell_{P}^{2}}{4 \pi}}\right)\left(\frac{1}{4} k_{B} N_{B H}\right)=-\frac{1}{2} M c^{2} \\
& T \delta S=\left(-\frac{\hbar}{2 \pi c k_{B}} \frac{G M}{\frac{N_{B H} \ell_{P}^{2}}{4 \pi}}\right)\left(\frac{1}{2} k_{B} N_{1}\right)=-\frac{N_{1}}{N_{B H}} M c^{2} \\
& -\frac{1}{2} M c^{2}=-\frac{N_{1}}{N_{B H}} M c^{2} \Leftrightarrow N_{1}=\frac{1}{2} N_{B H}
\end{aligned} .
$$

In other words, the event horizon can be regarded as a truly random message containing a balanced number of Planck area triangles encoding binary potential (8) equal to $-c^{2}$ or 0 . At $d=2 \sqrt{2}$ black hole provides gravitational potential (3) equal to $-c^{2} / 2$ at each vertex; but such a black hole is static, as all its Planck triangles are active. Only larger black holes such as $9 \pi$-bit black hole $(d=3)$ can at certain vertices have potential (3) larger than $-c^{2} / 2$ and thus are capable of generating a truly random message.

Another simple observation is that the average potential on the event horizon equals the event horizon potential at a vertex; that is

$$
\frac{\sum_{k=1}^{N_{1}}\left(-c^{2}\right)+\sum_{k=1}^{\left\lfloor N_{B H}\right\rfloor-N_{1}} 0}{N_{B H}}=\frac{-N_{1} c^{2}}{N_{B H}}=\frac{-\frac{1}{2} N_{B H} c^{2}}{N_{B H}}=-\frac{1}{2} c^{2} .
$$

$N_{B H}$-bit black hole provide $2^{\wedge}\left\lfloor N_{B H}\right\rfloor$ arrangements of Planck triangles. These arrangements can thus represent a set of addresses $a_{m}$ of vertices $m=1,2, \ldots, 2^{\wedge}\left\lfloor N_{B H}\right\rfloor$ of a unit $n$-cube (in units of $-c^{2}$ ) ordered in certain way (binary, Gray code, etc.). Then the following holds

$$
\frac{-\sum_{m=1}^{2^{\left\lfloor N_{B H}\right\rfloor}} a_{m}(i) c^{2}}{2^{\left\lfloor N_{B H}\right\rfloor}}=-\frac{1}{2} c^{2},
$$

where $i=1,2, \ldots,\left\lfloor N_{B H}\right\rfloor$ is $n$-cube coordinate. In other words the quotient of the sum of each triangle activations within the set of all triangle arrangements to the cardinality of 
this set equals the event horizon potential at a vertex. The meaning of this relation remains to be researched.

Perhaps all boundaries are topological spheres and differ only with radii and uniqueness or the rank of non-uniqueness (i.e. the number of cyclic spherical quadrilaterals) of the triangulation.

\section{Black hole blackbody radiation}

As wavelength measures a cycle $(2 \pi)$, it is reasonable to assume the minimum length of a cycle as Planck length $\left(\ell_{P}\right)$, as well as its natural multiplicity $\lambda=h / c m:=\ell \ell_{P}, l \in \mathbb{N}$. Thus Planck's law for blackbody spectral radiance

$$
B_{\lambda}(\lambda, T)=\frac{2 h c^{2}}{\lambda^{5}} \frac{1}{\exp \left(h c / \lambda k_{B} T\right)-1},
$$

plugging $\lambda:=\ell_{P}$ and black hole temperature (2) is

$$
B_{B H}(l, d)=\frac{2 h c^{2}}{l^{5} \ell_{P}^{5}} \frac{1}{\exp \left(4 \pi^{2} d / l\right)-1}
$$

for wavelength or

$$
B_{B H}\left(\frac{c}{l \ell_{P}}, d\right)=\frac{2 h c}{l^{3} \ell_{P}^{3}} \frac{1}{\exp \left(4 \pi^{2} d / l\right)-1}
$$

for frequency.

Black hole blackbody spectral radiance is almost like a bump function with measurable EMR $\left(\mathrm{W} \cdot \mathrm{sr}^{-1} \cdot \mathrm{m}^{-2} \cdot \mathrm{m}^{-1}\right)$ in a wavelength range of $10^{-12}$ to $10^{12}$ meter for a black hole of a size of an angstrom $(d=1 \mathrm{e} 25)$. Radiance of smaller holes vanishes for larger wavelengths; radiance of bigger ones vanishes for smaller wavelengths. Relations (14)-(16) are, as such, spacetime dimensional (they are derived under the assumption of a 2-sphere emitting blackbody radiation). The Bose-Einstein statistics term $1 /\left(\exp \left(4 \pi^{2} d / l\right)-1\right)$ remains fractionally dimensional.

\section{Black Hole Diameter Fluctuations}

Black hole absorption was studied by Jacob Bekenstein [9] to derive his famous bound on black hole entropy. Emission was shown by Steven Hawking [20] to be responsible for micro black holes collapses. Taking both processes together the black hole mass and thus also its diameter fluctuates in time.

Informationless black hole absorption $[9,19]$ and emission [8] formulas for a subatomic particle having a wavelength corresponding to the black hole radius can be extended (cf. Appendices) to arbitrary wavelengths $l$

$$
N_{A / E}(d, l)=64 \pi^{3} \frac{1}{l^{2}} \pm 16 \pi^{2} \frac{d}{l}+\pi d^{2},
$$

which can be described more compactly $\left(N_{B H}=\pi d^{2}\right)$ as a recurrence relation

$$
d_{k+1}^{A / E}=\sqrt{64 \pi^{2} \frac{1}{l^{2}} \pm 16 \pi \frac{d_{k}}{l}+d_{k}^{2}},
$$

describing new diameter $d_{k+1}$ of a black hole (2-sphere) having an initial diameter $d_{k}$ after absorbing $(A, "+")$ or emitting $(E, "-")$ a wavelength $l$. Thus 2D black hole collapses 
$\left(d_{k+1} E=0\right)^{3}$ after emission of the wavelength $l=8 \pi / d_{k}$, while its diameter does not change $\left(d_{k+1} E=d_{k}\right)$ iff $l=4 \pi / d_{k}$, as illustrated in Fig. 2.
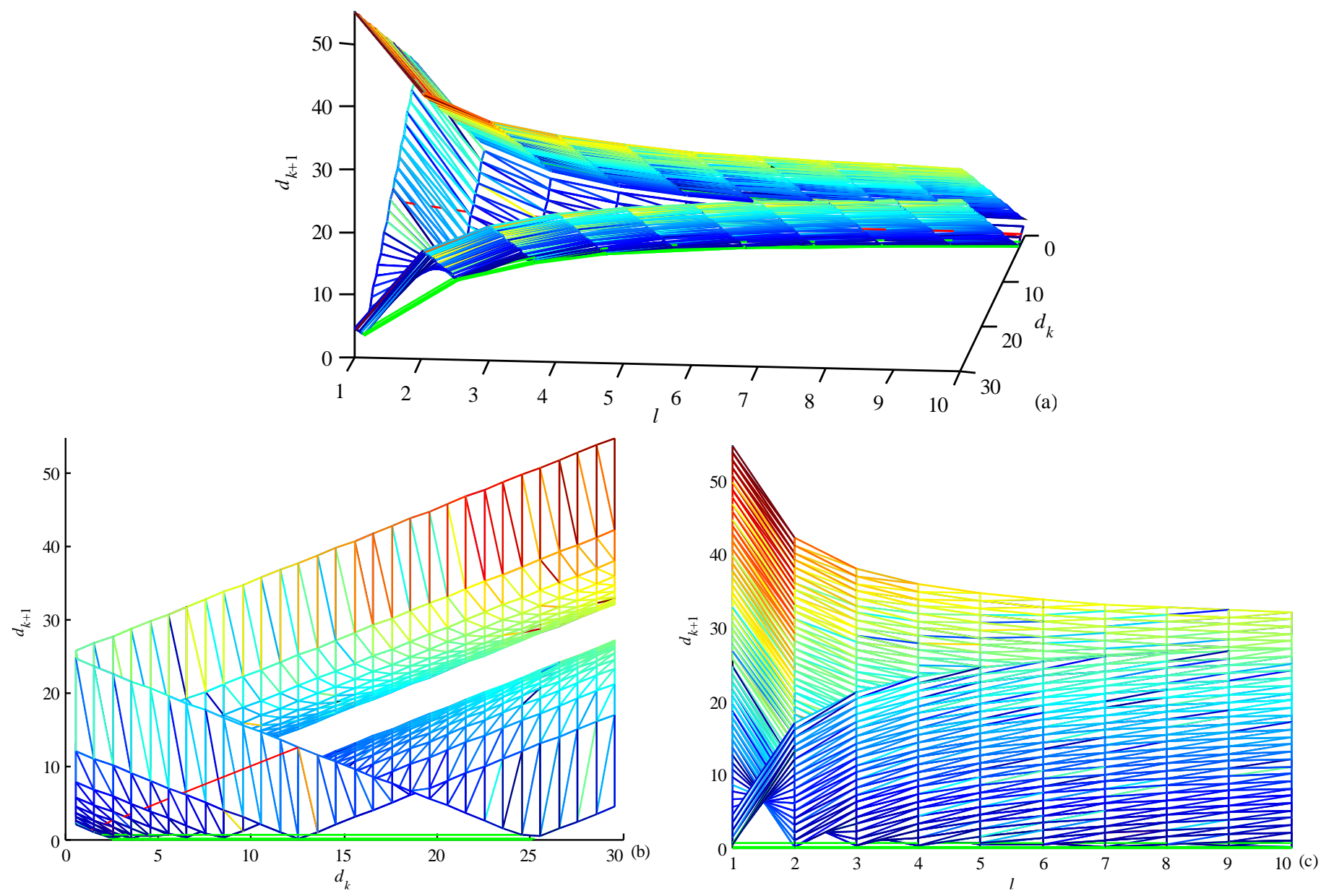

Fig. 2: New 2-sphere black hole diameter $d_{k+1}$ after absorption (a-c) and emission (b, c) of a particle having wavelength $l$. Red curve represents constant diameter, green ones - black hole collapse. Sinusoidal characteristic of collapse is due to $l \in \mathbb{N}$.

This derivation can be taken to other dimensions yielding the following recurrence relation for black hole emission ${ }^{4}$

$$
\left(d_{k+1}^{E}\right)^{n-1}=\left(d_{k}-\frac{8 \pi}{l}\right)^{n-1} .
$$

Thus all black holes collapse after emission of a wavelength close to $l=8 \pi / d_{k}$, while their diameter does not change $\left(d_{k+1} E=d_{k}\right)$ after emission of wavelengths listed in Table 2 .

Analytical formula $d_{k+1} E(n)$ for constant diameter emission in any dimension, if one exists, remains to be researched. Interestingly odd dimensionalities admit real wavelength $l=4 \pi / d_{k}$. In even dimensionalities all constant diameter wavelengths are complex and for $n>4$ complicated.

$31 / 2 \pi\left(T_{P}\right)$ or $1 / \sqrt{ } \pi$ (bit) is more viable threshold of collapse.

4 and absorption with "+" instead of " -". 
Table 2: Black hole constant diameter after emission of a wavelength $l$ in dimensions $n=2,3, \ldots, 9$.

\begin{tabular}{|l|l|}
\hline$n$ & $\left(\mathrm{~d}_{k+1}\right)^{n-}$ \\
\hline 2 & no solution for $l \neq \infty$ \\
\hline 3 & $l=4 \pi / d_{k}$ \\
\hline 4 & $l_{1,2}=\frac{4 \pi}{d_{k}}\left(1 \pm \frac{\sqrt{3}}{3} i\right)$ \\
\hline 5 & $l_{1}=\frac{4 \pi}{d_{k}}, l_{2,3}=\frac{4 \pi}{d_{k}}(1 \pm i)$ \\
\hline 7 & $l_{1,2,3,4}=\frac{4 \pi}{d_{k}}\left(1 \pm \frac{\sqrt{5 \pm 2 \sqrt{5}}}{\sqrt{5}} i\right)$ \\
\hline 8 & $l_{1}=\frac{4 \pi}{d_{k}}, l_{2,3}=\frac{4 \pi}{d_{k}}(1 \pm \sqrt{3} i), l_{4,5}=\frac{4 \pi}{d_{k}}\left(1 \pm \frac{\sqrt{3}}{3} i\right)$ \\
\hline 9 & $\begin{array}{l}l_{1}=\frac{4 \pi}{d_{k}}, l_{2,3,4,5}=\frac{4 \pi}{d_{k}}(1 \pm i \sqrt{3 \pm 2 \sqrt{2}}) \\
l_{6,7}=\frac{4 \pi}{d_{k}}(1 \pm i)\end{array}$ \\
\hline
\end{tabular}

\section{Black Holes Quantum Statistics}

If a black hole Planck triangle emits/absorbs a photon (boson), as it changes its state from active to inactive or vice versa and an electron (fermion) energy level is respectively decreased/increased in this interaction, then the event horizon can be described by Bose-Einstein (BE) and Fermi-Dirac (FD) statistics with degeneracy interpreted as the number of Planck triangles $\left\lfloor\pi d^{2}\right\rfloor$ on the event horizon. No Planck triangle is distinct and each one is equally capable of emitting/absorbing a subatomic particle having energy corresponding to wavelength $l$. There are certainly no dedicated microwave or X-ray triangles on the horizon. These spacetime dimensional (due to $\left\lfloor\pi d^{2}\right\rfloor$ term) statistics along with the Maxwell-Boltzmann ${ }^{5}(\mathrm{MB})$ one are thus as follows

$$
\begin{gathered}
N_{B E}(d, l)=\frac{\left\lfloor\pi d^{2}\right\rfloor}{e^{4 \pi^{2} d / l}-1}, \\
N_{M B}(d, l)=\frac{\left\lfloor\pi d^{2}\right\rfloor}{e^{4 \pi^{2} d / l}}, \\
N_{F D}(d, l)=\frac{\left\lfloor\pi d^{2}\right\rfloor}{e^{4 \pi^{2} d / l}+1},
\end{gathered}
$$

as illustrated in Fig. 3, where $N(d, l)$ is the average number of bosons, classical particles, and fermions, respectively, having wavelength $l$.

${ }^{5} \mathrm{MB}$ statistics lies between BE and FD statistics. 


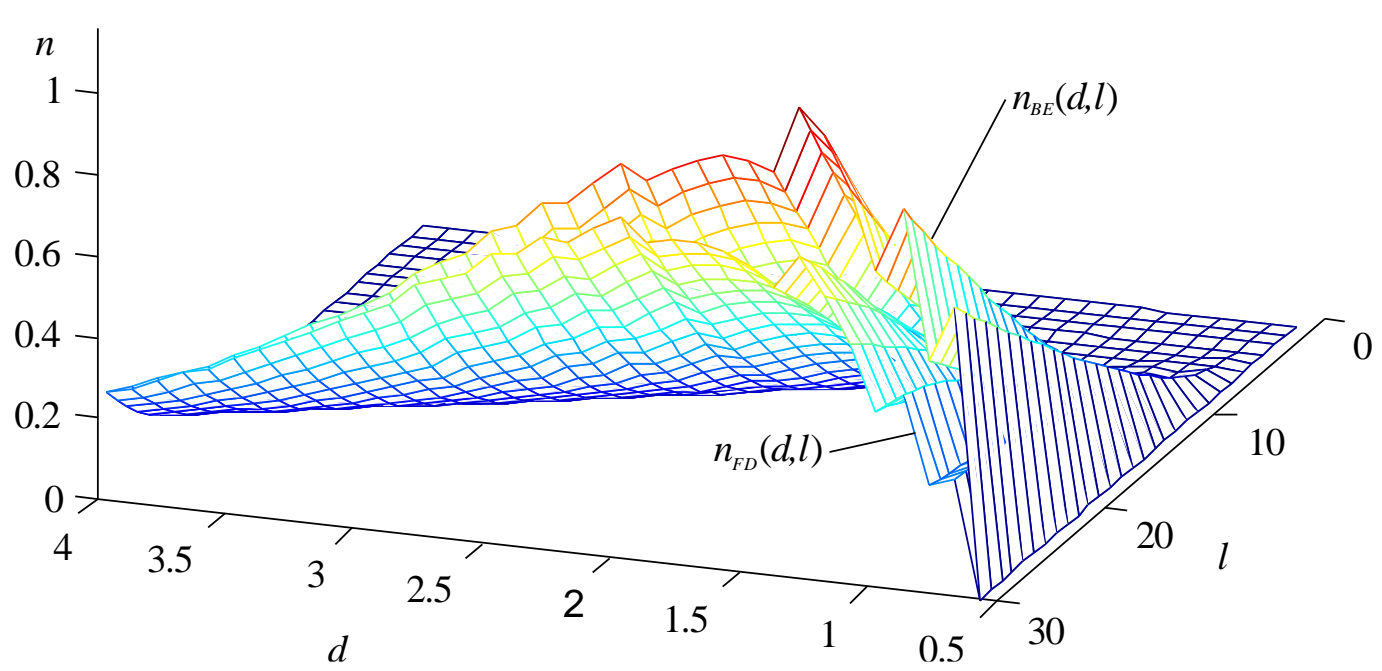

Fig. 3: Bose-Einstein (BE, top) and Fermi-Dirac (FD, bottom) black hole statistics.

The nontrivial black hole microstate degeneracy starts at 3 vertices [21] of the Voronoi triangulation that corresponds to the $\pi$-bit black hole with 4 vertices.

For $N>0$ photons to be emitted/absorbed or $N>0$ electron energy levels to be respectively decreased/increased on average, $N(d, l) \geq N$ and the following bounds, illustrated in Fig. 4 for $N=1$, are obtained from statistics (20)-(22) 6

$$
\begin{gathered}
l_{B E}(d, N) \geq \frac{4 \pi^{2} d}{\ln \left(\pi d^{2}+N\right)-\ln (N)}, \\
l_{M B}(d, N) \geq \frac{4 \pi^{2} d}{\ln \left(\pi d^{2}\right)-\ln (N)}, \\
l_{F D}(d, N) \geq \frac{4 \pi^{2} d}{\ln \left(\pi d^{2}-N\right)-\ln (N)} .
\end{gathered}
$$

Each bound has the singularity

$$
d_{B E}^{\mathrm{sin}}=0, d_{M B}^{\mathrm{sin}}= \pm \sqrt{\frac{N}{\pi}}, d_{F D}^{\mathrm{sin}}= \pm \sqrt{\frac{2 N}{\pi}},
$$

and minimum

$$
d_{B E}^{\min }= \pm \sqrt{-N\left(\frac{W_{0}\left(-2 e^{-2}\right)+2}{\pi W_{0}\left(-2 e^{-2}\right)}\right)}, d_{M B}^{\min }= \pm e \sqrt{\frac{N}{\pi}}, d_{F D}^{\min }= \pm \sqrt{N \frac{W_{0}\left(2 e^{-2}\right)+2}{\pi W_{0}\left(2 e^{-2}\right)}}
$$

where $W_{0}(x)$ is the Lambert $W$ function (the omega function). Minima are listed in Table 3 along with corresponding minimum wavelengths.

Negative wavelengths for positive diameters can be interpreted in terms of emission/absorption, with positive wavelengths representing black hole absorption and negative wavelengths representing black hole emission. BE bound (23), having no negative wavelengths, illustrates that bosons (photons) cannot escape from a black hole. If we demand the Compton wavelength to be larger than Planck length then the following equivalences hold [8]

${ }^{6} n \leq\lfloor x\rfloor \boxminus n \leq x$ (property of the floor function). 


$$
\begin{gathered}
\hbar=\frac{\hbar}{m c}>\ell_{P}=\sqrt{\frac{\hbar G}{c^{3}}} \Leftrightarrow m<\sqrt{\frac{\hbar c}{G}}=m_{P}, \\
\lambda=\frac{h}{m c}>\ell_{P}=\sqrt{\frac{\hbar G}{c^{3}}} \Leftrightarrow m<2 \pi m_{P},
\end{gathered}
$$

where the latter equation is satisfied by $64 \pi^{3}$-bit black hole (cf. Table 1 ).

Table 3: BE, MB, and FD bounds for $N=1,2, \ldots, 6$.

\begin{tabular}{|l|l|l|l|l|l|l|}
\hline$N$ & $d^{\min _{B E}}$ & $l^{\min _{B E}}$ & $d^{\min _{M B}}$ & $l^{\min _{M B}}$ & $d^{\min _{F D}}$ & $l^{\min _{F D}}$ \\
\hline 1 & 1.1173 & 27.678 & 1.5336 & 30.273 & 1.8007 & 32.054 \\
\hline 2 & 1.5800 & 39.142 & 2.1689 & 42.812 & 2.5465 & 45.332 \\
\hline 3 & 1.9352 & 47.939 & 2.6563 & 52.434 & 3.1188 & 55.520 \\
\hline 4 & 2.2345 & 55.355 & 3.0673 & 60.545 & 3.6013 & 64.109 \\
\hline 5 & 2.4982 & 61.889 & 3.4293 & 67.692 & 4.0264 & 71.676 \\
\hline 6 & 2.7367 & 67.796 & 3.7566 & 74.152 & 4.4107 & 78.517 \\
\hline
\end{tabular}
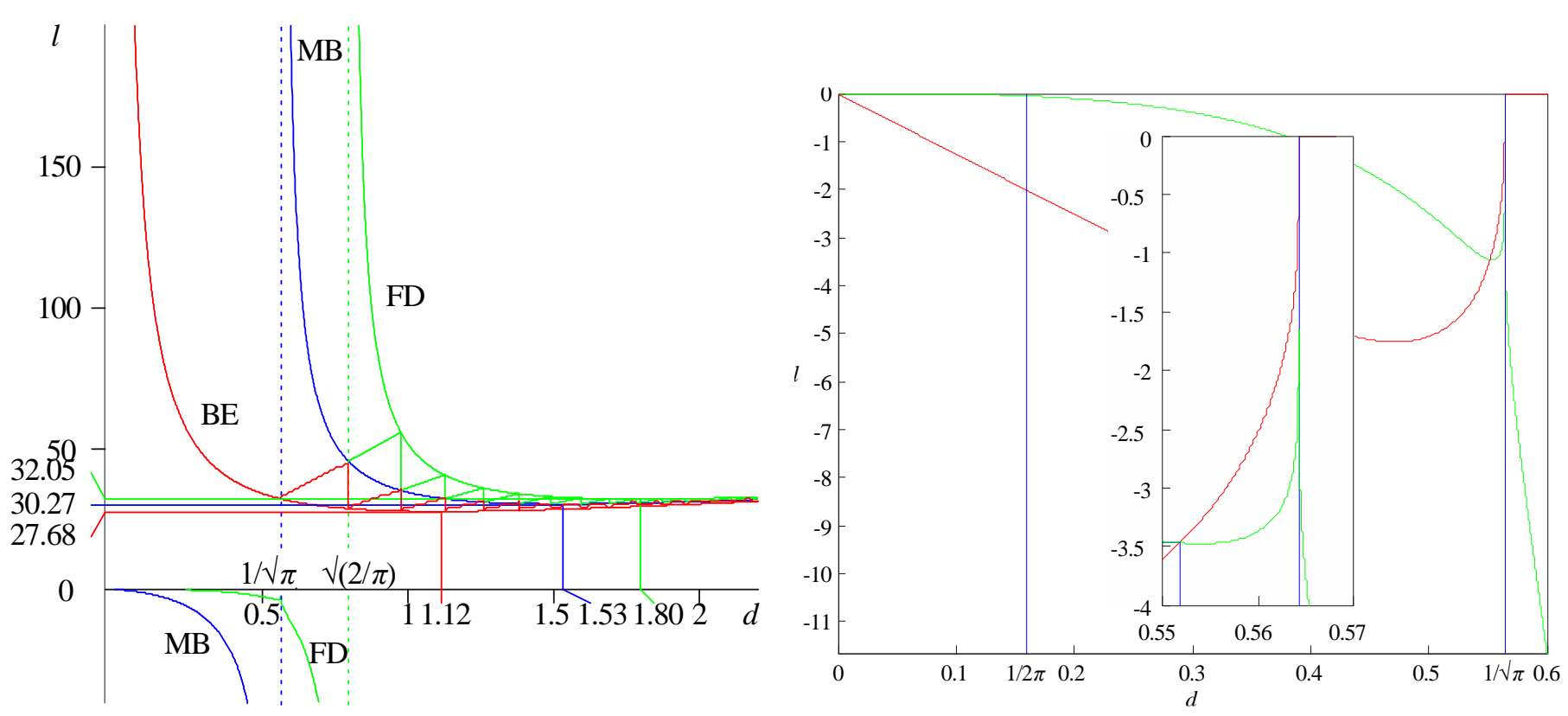

Fig. 4: (a) Wavelength bounds for BE (red), MB (blue), and FD (green) statistics for Delaunay triangulated black hole as a function of diameter/dimensionality $d$ with $N=1$. (b) Real (green) and imaginary (red) part of the wavelength bound for FD statistics as a function of $d$ in the vicinity of $1 / \sqrt{ } \pi$.

For $0<d \leq \sqrt{ }(N / \pi)$ wavelengths $l$ given by FD bound (25) are complex, as shown in Fig. 4(b). Below this threshold, which corresponds to the diameter of 1-bit black hole, black hole emits complex wavelengths and only after exceeding this diameter they become real and thus observable. Perhaps only negative and real wavelengths in the FD bound (25) within the range $\sqrt{ }(N / \pi)<d \leq \sqrt{ }(2 N / \pi)$ represent Hawking radiation of fermions. Interestingly the real part of the wavelength below $d=1 /(2 \pi)$ Planck temperature threshold vanishes.

The logistic function

$$
f_{F D}(d)=\frac{1}{e^{-\mu d}+1}=\frac{e^{\mu d}}{e^{\mu d}+1},
$$

where $\mu$ determines its steepness has derivative 


$$
f_{F D}^{\prime}(d)=\mu f_{F D}(d)\left[1-f_{F D}(d)\right]=\frac{\mu}{4 \cosh ^{2}(\mu d / 2)},
$$

which is the continuous version of the logistic map

$$
x_{n+1}^{F D}=\mu x_{n}^{F D}\left(1-x_{n}^{F D}\right),
$$

having a bifurcation diagram shown in Fig. 5(a). For certain values of $\mu$ within the range of $-2 \leq \mu \leq 4$ [22] (for $\mu<-2$ and $\mu>4$ (32) is divergent), in particular for $-2 \leq \mu \leq-1$ and $3 \leq \mu \leq 4$, the logistic map displays intermittent (irregular alternation of periodic and chaotic dynamics) behavior.
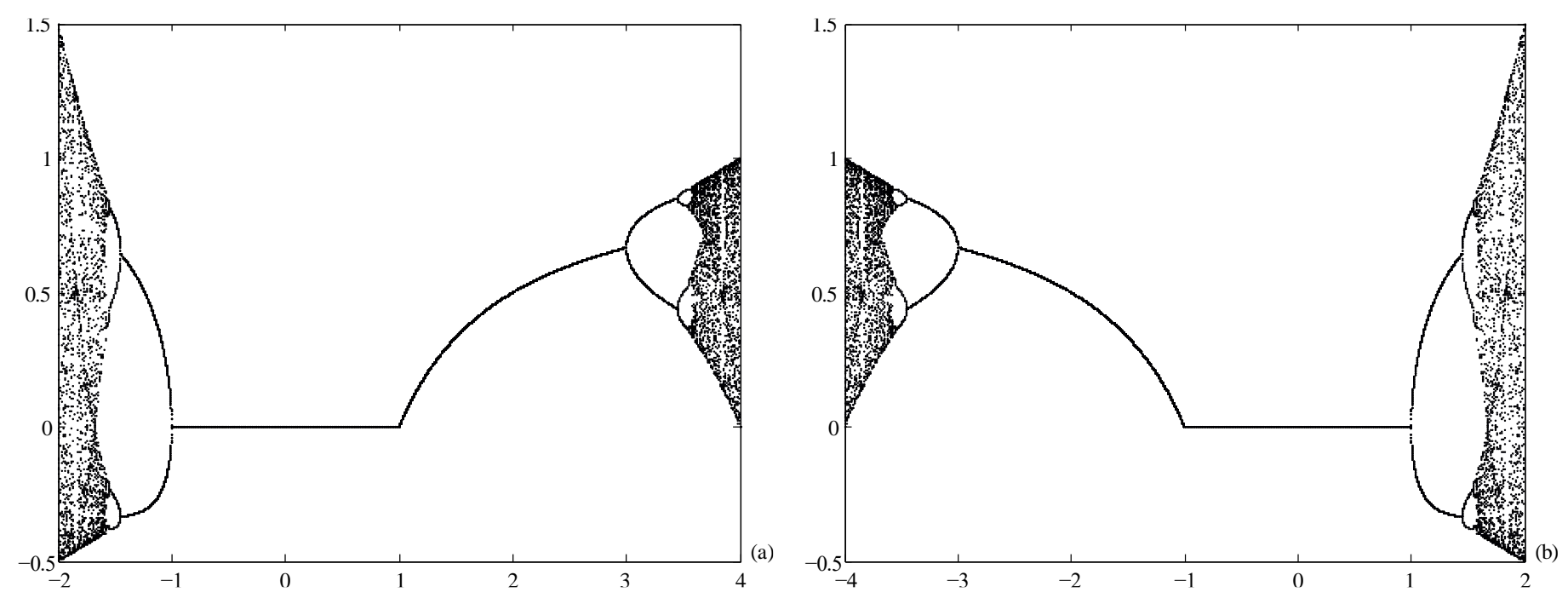

Fig. 5: Bifurcation diagrams of the logistic maps corresponding to FD (a) and BE statistics (b).

A similarity of the logistic function and FD statistics is apparent (hence the index $F D$ ). Comparing FD statistics (22) with the logistic function (30) we conclude that

$$
\mu=\frac{4 \pi^{2}}{l}
$$

with $\max (\mu)=4 \pi^{2} \approx 39.4784$ (for $l=1$ ) and

$$
e^{\mu d}=\left\lfloor\pi d^{2}\right\rfloor
$$

plugging (33) to (34) and solving for $l$ leads to

$$
l_{F D}(d)=\frac{4 \pi^{2} d}{\ln \left(\left\lfloor\pi d^{2}\right\rfloor\right)}
$$

the equation resembling wavelength bound for MB statistics (24). A similar relation

$$
l_{B E}(d)=\frac{4 \pi^{2} d}{\ln \left(\left\lfloor\pi d^{2}\right\rfloor+1\right)}
$$

can by postulated for wavelength bound for BE statistics (23). This leads to the BE logistic function 


$$
f_{B E}(d)=\frac{1}{1-e^{-\mu d}}=\frac{e^{\mu d}}{e^{\mu d}-1}
$$

having derivative

$$
f_{B E}^{\prime}(d)=\mu f_{B E}(d)\left[f_{B E}(d)-1\right]=\frac{-\mu}{4 \sinh ^{2}(\mu d / 2)}
$$

which is the continuous version of the BE logistic map

$$
x_{n+1}^{B E}=\mu x_{n}^{B E}\left(x_{n}^{B E}-1\right)
$$

having a bifurcation diagram shown in Fig. 5(b). It displays intermittent behavior for $-4 \leq \mu \leq-3$ and $1 \leq \mu \leq 2$ and is divergent for $\mu<-4$ and $\mu>2$. Obviously (32) turns into (39) by swapping $\mu$ with $-\mu$. Negative $\mu$ by virtue of (33) corresponds to negative wavelengths.

Relations (35) and (36) illustrated in Fig. 4(a) have form of a sawtooth waves. The BE wave (36) bounces between the BE wavelength bound (23) and the MB wavelength bound (24), while the FD wave (35) bounces between the MB wavelength bound (24) and the FD wavelength bound (25). Checking intermittent values of the parameter $\mu$ in maps (32) and (39) in dependence of $l$ given by (33) yields

$$
3 \leq \mu \leq 4 \quad \Leftrightarrow \quad \pi^{2} \leq l \leq \frac{4}{3} \pi^{2}
$$

for the FD logistic map (32) and

$$
1 \leq \mu \leq 2 \quad \Leftrightarrow \quad 2 \pi^{2} \leq l \leq 4 \pi^{2}
$$

for the BE logistic map (39).

Convergence bounds of the maps (32) and (39) are related: $(-2)^{2}=4$ and $(2 i)^{2}=-4$. On the other hand the logistic function (30) has codomain $0<f_{F D}<1$, while the BE logistic function (37) has codomain $-\infty<f_{B E}<0$ and $1<f_{B E}<\infty$, which renders range $(0,1)$ distinct to the range $(-1,0)$. A similar asymmetry has been observed for graphene [23] with regard to the positive and negative number of layers. Perhaps exotic $\mathbb{R}^{4}$ property of the Euclidean space $\mathbb{R}^{4}$ applies solely to $\mathbb{R}^{4}$ not to $\mathbb{R}^{-4}, \mathbb{R}^{4 i}$, or $\mathbb{R}^{-4 i}$. Volume of unit radius (-1)-ball equals $1 / \pi \quad$ [24].

Black hole interaction with the environment described in this way certainly requires further research taking into account the imaginary set of Planck units [25].

\section{Observer as a Dissipative Structure}

Fundamental thermodynamic equilibriums of nature are black holes emitting informationless blackbody radiation characterized by zero Gibbs free energy and representing the degenerate case of relativistic Bose-Einstein statistics (20) [26]. Informationless nature of blackbody radiation is further asserted by the no-hiding theorem [27]. The existence of non-degenerate radiation is responsible for the departure from blackbody radiation met with in astrophysical studies [26]. Certainly stars are also dissipative structures.

A dissipative structure is enclosed by non-uniquely defined Delaunay triangulated boundary. This allows the dissipative structure to use the additional bits provided by cyclic geodesic quadrilaterals to locally decrease entropy, staying away of thermodynamic equilibrium. Obviously such quadrilaterals are scarce but this is confirmed by scarceness of dissipative structures such as planets, stars, hurricanes, living organisms, etc. in observable nature. Dissipative structure is defined by non-uniquely defined Delaunay triangulated surface, while systems in thermodynamic equilibrium, such as black holes event horizons, are defined by uniquely defined Delaunay triangulated bounda- 
ries. A relation between non-uniqueness of the triangulation for cyclic geodesic quadrilaterals and zero weights of the cotan Laplacian only for geodesic rectangles requires further research. Any one of the two possible triangulations on 4 vertices of a rectangle produces the same cotan Laplacian [6]. Yet there exist isospectral but non-isometric manifolds.

Boundary of perception of living organisms is 2-dimensional, a gastrointestinal tract requires at least 3-dimensions and time to be formed [28]. An observer (a human being in particular) is a dissipative structure [18] that operates out of thermodynamic equilibrium. Black holes may exist in 2-dimensions but living cells cannot.

\section{Discussion}

The findings of this paper should perhaps be used in an improved model of a black hole interaction with the environment that remains to be researched. It would certainly provide further insight into the sonoluminescence phenomenon and instability of quantum black holes.

Blackbody radiation carries no information and depends only on temperature of a radiating object. This informationless feature relates blackbody radiation with a truly random message of $\left\{0,-c^{2}\right\}$ (11). For black holes blackbody radiation spectrum is determined solely by its informational capacity (size) (2). This size dependence is uneasy. Fortunately black holes are not the only observable objects that emit blackbody radiation. Neutron stars supported against collapse by neutron degeneracy pressure and white dwarfs supported by electron degeneracy pressure, both pressures due to the Pauli exclusion principle, also emit blackbody radiation. Thus they also should have unique Delaunay triangulations and also generate truly random messages; a thermodynamic equilibrium blackbody radiation.

Funding: This research received no external funding.

Acknowledgments: I thank my wife for inspiration; Mirek (who found that black holes invalidate Jordan-Brouwer separation theorem), Rajmund, and Łukasz for helpful discussions and encouragement; and Wawrzyniec for reviewing this paper.

Conflicts of Interest: The author declares no conflict of interest.

\section{References}

[1] Ludwig Boltzmann, "Über die Beziehung zwischen dem zweiten Hauptsatze des mechanischen Wärmetheorie und der Wahrscheinlichkeitsrechnung, respective den Sätzen über das Wärmegleichgewicht” Von dem c. M. Ludwig Boltzmann in Graz Sitzb. d. Kaiserlichen Akademie der Wissenschaften, mathematich-naturwissen Cl. LXXVI, Abt II, 1877, pp. 373-435., av. at http://users.polytech.unice.fr/ leroux/boltztrad.pdf.

[2] Thomas S. Kuhn, “Black-Body Theory and the Quantum Discontinuity, 1894-1912”. Oxford University Press, 1978.

[3] Pierre Teilhard de Chardin, “The Phenomenon of Man”. Paris, Éditions du Seuil. 1955.

[4] Mathieu Desbrun, Eva Kanso, Yiying Tong, "Discrete Differential Forms for Computational Modeling”. Discrete Differential Geometry. Oberwolfach Seminars, vol 38. Birkhäuser Basel (2008).

[5] John Archibald Wheeler, "Proc. 3rd Int. Symp. on Foundations of Quantum Mechanics". p.354 (Phys. Soc. Japan (1990), Tokyo, 1989).

[6] Szymon Łukaszyk, "Four Cubes". arXiv:2007.03782 [math.GM], 7 Jul 2020.

[7] Benoit B. Mandelbrot, "Negative Fractal Dimensions And Multifractals”. Physica A 163 (1990) 306-315.

[8] Szymon Łukaszyk, "Black hole horizons as binary messages”. arXiv:1910.11081 [physics.gen-ph], 15 Feb 2021.

[9] J.D. Bekenstein, "Black holes and entropy”. Phys. Rev. D 7 (1973) 2333 (http://www.scholarpedia.org/article/Bekenstein bound, http://www.scholarpedia.org/article/Bekenstein-Hawking entropy).

[10] Erik Verlinde, "On the Origin of Gravity and the Laws of Newton". Journal of High Energy Physics, doi:10.1007/JHEP04(2011)029, arXiv:1001.0785v1 [hep-th] 6 Jan 2010. 
[11] Edward Charles Titchmarsh, David Rodney Heath-Brown, “The Theory of the Riemann Zeta-function (2 ${ }^{\text {nd }}$ ed.)”. Oxford: Oxford U. P., ISBN 0-19-853369-1.

[12] Max Wardetzky, “Generalized Barycentric Coordinates in Computer Graphics and Computational Mechanics". Chapter 5, A Primer on Laplacians, CRC Press, 2017.

[13] Samuel. Rippa, "Minimal roughness property of the Delaunay triangulation”. Comput. Aided Geom. Design 7, 489-497, 1990.

[14] Renjie Chen, Yin Xua, Craig Gotsman, Ligang Liu, "A spectral characterization of the Delaunay triangulation”. Computer Aided Geometric De-sign, Elsevier Vol. 27, Issue 4, May 2010.

[15] Alexander I. Bobenko, Boris A. Springborn, “A Discrete Laplace-Beltrami Operator for Simplicial Surfaces”. Discrete Comput Geom (2007) 38: 740-756.

[16] Sabine Hossenfelder, "Comments on and Comments on Comments on Verlinde's paper "On the Origin of Gravity and the Laws of Newton". arXiv:1003.1015v1 [gr-qc] 4 Mar 2010.

[17] Veronica G. Vergara Larrea, "Construction of Delaunay Triangulations on the Sphere: A Parallel Approach". Florida State University Libraries, Electronic Theses, Treatises and Dissertations, 2011.

[18] Ilya Prigogine, Isabelle Stengers, “Order Out of Chaos: Man's New Dialogue with Nature”. Bantam Books, April 1984.

[19] Leonard Susskind, "Black Hole War: My Battle with Stephen Hawking to Make the World Safe for Quantum Mechanics". Little, Brown and Company, July 7, 2008.

[20] Stephen Hawking, "Black hole explosions?”. Nature. 248 (5443): 30-31. 1974 (http://www.scholarpedia.org/article/Hawking_radiation).

[21] Aharon Davidson, "From Planck area to graph theory: Topologically distinct black hole microstates". Phys. Rev. D 100, 081502(R) - Published 18 October 2019

[22] Tsuchiya, Takashi; Yamagishi, Daisuke, "The Complete Bifurcation Diagram for the Logistic Map". Z. Naturforsch. 52a: 513-516. 11 Feb 1997.

[23] Szymon Łukaszyk, “A short note about the geometry of graphene”. DOI: 10.13140/RG.2.2.11500.18569, November 2020.

[24] Szymon Łukaszyk, “A simple recursive formula for volumes and surfaces of n-balls”. DOI: 10.13140/RG.2.2.14751.38566, May 2020.

[25] Szymon Łukaszyk, “A short note about graphene and the fine structure constant”. DOI: 10.13140/RG.2.2.21718.88641, October 2020.

[26] Kothari, D. S., and B. N. Singh, "Bose-Einstein Statistics and Degeneracy". Proceedings of the Royal Society of London. Series A, Mathematical and Physical Sciences, vol. 178, no. 973, 1941, pp. 135-152.

[27] Samuel L. Braunstein and Arun K. Pati, "Quantum Information Cannot Be Completely Hidden in Correlations: Implications for the Black-Hole Information Paradox”. Phys. Rev. Lett. 98, 080502 -23 February 2007.

[28] Michio Kaku, "Hyperspace: A Scientific Odyssey Through Parallel Universes, Time Warps, and the 10th Dimension”. ISBN 0-19-286189-1. 1 Feb 1995.

\section{Appendices}

1. Derivation of black hole temperature (2):

$$
\begin{aligned}
& T=\frac{T_{P}}{2 \pi d}=\frac{T_{P}}{2 \pi} \frac{\ell_{P}}{2 R_{B H}}=\frac{T_{P} \ell_{P}}{4 \pi} \frac{c^{2}}{2 G M}= \\
& =\frac{c^{2}}{8 \pi G M} \sqrt{\frac{\hbar c^{5}}{G k_{B}^{2}}} \sqrt{\frac{\hbar G}{c^{3}}}=\frac{\hbar c^{3}}{8 \pi G k_{B} M}
\end{aligned} .
$$

2. Another form of Planck-Einstein relation: 


$$
\begin{aligned}
& E=h v=h \frac{c}{\lambda}=\frac{h c}{l \ell_{P}}=\frac{2 \pi \hbar c}{l} \sqrt{\frac{c^{3}}{\hbar G}}= \\
& =\frac{2 \pi}{l} \frac{k_{B}}{k_{B}} \sqrt{\frac{\hbar^{2} c^{2} c^{3}}{\hbar G}}=\frac{2 \pi}{l} k_{B} T_{P}
\end{aligned},
$$

3. Derivation of black hole diameter fluctuations.

After absorbing/emitting a subatomic particle having a wavelength $l 2$-sphere black hole diameter defined by its mass increases(+)/decreases(-) by the Compton mass $m=h /(c \lambda)$ of this particle. Thus we arrive at

$$
\begin{aligned}
& D^{A / E}=D \pm \delta D=d \ell_{P} \pm \frac{4 G}{c^{2}} \frac{2 \pi \hbar}{c l \ell_{P}} \\
& =d \ell_{P} \pm \frac{8 \pi \hbar G}{l c^{3} \ell_{P}}=d \ell_{P} \pm \frac{8 \pi \ell_{P}}{l}=(d \pm 8 \pi / l) \ell_{P}
\end{aligned}
$$

Accordingly black hole area increases/decreases as well

$$
\begin{gathered}
A^{A / E}=\pi\left(d^{A / E}\right)^{2} \ell_{P}^{2}=\pi\left(d \ell_{P} \pm \frac{8 \pi G \hbar}{c^{3} l \ell_{P}}\right)^{2} \\
\pi\left(d^{A / E}\right)^{2} \ell_{P}^{2}=\pi\left(d \ell_{P} \pm \frac{8 \pi}{l} \ell_{P}\right)^{2}= \\
=\pi \ell_{P}^{2}\left(d \pm \frac{8 \pi}{l}\right)^{2} \\
\left(d_{k+1}^{A / E}\right)^{2} \ell_{P}^{2}=d_{k}^{2} \ell_{P}^{2} \pm 2 d_{k} \ell_{P} \frac{8 \pi G \hbar}{c^{3} l \ell_{P}}+\frac{64 \pi^{2} G^{2} \hbar^{2}}{c^{6} l^{2} \ell_{P}^{2}} \\
\left(d_{k+1}^{A / E}\right)^{2}=d_{k}^{2} \pm \frac{16 \pi d_{k} G \hbar}{c^{3} l \ell_{P}^{2}}+\frac{64 \pi^{2} G^{2} \hbar^{2}}{c^{6} l^{2} \ell_{P}^{4}}= \\
=d_{k}^{2} \pm \frac{16 \pi d_{k} G \hbar}{c^{3} l} \frac{c^{3}}{\hbar G}+\frac{64 \pi^{2} G^{2} \hbar^{2}}{c^{6} l^{2}} \frac{c^{6}}{\hbar^{2} G^{2}}= \\
=d_{k}^{2} \pm 16 \pi \frac{d_{k}}{l}+64 \pi^{2} \frac{1}{l^{2}}
\end{gathered}
$$

If $l=d / 2[9,19]$ then

$$
d_{k+1}^{A / E}= \pm \sqrt{256 \pi^{2} \frac{1}{d_{k}^{2}} \pm 32 \pi+d_{k}^{2}} .
$$

4. Derivation of Landauer black hole diameter 


$$
\begin{aligned}
& M_{B H} c^{2}=T k_{B} \ln (2) \\
& \frac{d \ell_{P} c^{2}}{4 G} c^{2}=\frac{T_{P}}{2 \pi d} k_{B} \ln (2) \\
& \frac{d c^{4}}{4 G} \sqrt{\frac{\hbar G}{c^{3}}}=\sqrt{\frac{\hbar c^{5}}{G k_{B}^{2}}} \frac{k_{B} \ln (2)}{2 \pi d} \\
& \frac{d}{2}=\frac{\ln (2)}{\pi d} \quad d= \pm \sqrt{\frac{\ln (4)}{\pi}} \approx \pm 0.6643
\end{aligned}
$$

5. Black hole diameter after absorption or emission of a wavelength $l$ in dimensions $n=2$, $3, \ldots, 5$.

\begin{tabular}{|l|l|}
\hline$n$ & $\left(\mathrm{~d}_{k+1}{ }^{A / E}\right)^{n-1}$ \\
\hline 2 & $d_{k} \pm 8 \pi / l$ \\
\hline 3 & $d k^{2} \pm 16 \pi d_{k} / l+64 \pi^{2} / l^{2}$ \\
\hline 4 & $d k^{3} \pm 24 \pi d k^{2} / l+192 \pi^{2} d_{k} / l^{2} \pm 512 \pi^{3} / l^{3}$ \\
\hline 5 & $d k^{4} \pm 32 \pi d k^{3} / l+384 \pi^{2} d k^{2} / l^{2} \pm 2048 \pi^{3} d_{k} / l^{3}+4096 \pi^{4} / l^{4}$ \\
\hline
\end{tabular}

